\title{
The Influence of Soft Vibrational Modes on our Understanding of Oxide Surface Structure
}

\author{
N. M. Harrison, X.-G. Wang*, J. Muscat ${ }^{\dagger}$ and M. Scheffler* \\ CCLRC Daresbury Laboratory, Daresbury, Warrington, WA4 4AD, U.K. \\ * Fritz-Haber-Institut der Max-Planck Gesellschaft, Faradayweg 4-6, D-14195 Berlin-Dahlem, \\ Germany. \\ $\dagger$ Present address: CSIRO Minerals, Box 312, Clayton South, Victoria 3169, Australia.
}

\begin{abstract}
We examine the reasons for the poor quantitative agreement between the structures predicted from the minimum energy configuration of first principles calculations and those deduced from surface xray diffraction experiments for the structure properties of the $\mathrm{TiO}_{2}(110)$ surface. In order to confine all numerical approximations very large scale all-electron first principles calculations are used. We find a very soft, anisotropic and anharmonic surface rigid-unit vibrational mode which involves displacements of the surface ions of approximately $0.15 \AA$ for thermal vibrations corresponding to room temperature. It is concluded that in order to perform an accurate comparison between theory and experiment for this and perhaps other oxide surfaces it will be necessary to take account of such anisotropic vibrations in models used to interpret experimental data. In addition the contribution of the vibrational entropy to the surface free energy is likely to be significant and must be taken into account when computing surface energies and structures.
\end{abstract}




\section{Introduction}

Titanium dioxide $\left(\mathrm{TiO}_{2}\right)$ is an interesting and industrially important material which has been studied extensively in recent years. This interest is, in part, due to the existing applications of $\mathrm{TiO}_{2}$ as a white pigment and as a catalyst support $[1,2]$ but also due to the many new applications currently under investigation. Recent examples include self cleaning paint coatings [3], catalytically active paving stones [4], solar cells [5] and water disinfection [6]. $\mathrm{TiO}_{2}$ is also of interest as a model transition metal oxide. It is readily reduced in the bulk and at the surfaces resulting in the occupation of $\mathrm{Ti} d$ orbitals which has a profound effect on the physical and electronic structure $[7,8,9]$. However, the bulk structure of $\mathrm{TiO}_{2}$ is simple relative to many oxides and thus a variety of empirical and first principles theories can readily be used to compute its physical and chemical properties. It is therefore an excellent model system displaying many of the properties of more complex oxides which can be studied relatively easily using a variety of experimental and theoretical techniques.

Many of the important and useful properties of $\mathrm{TiO}_{2}$ depend on the physical and electronic structure of its surfaces. The structure of the most stable (110) surface has attracted enormous interest in recent years. Experimental studies have included low energy electron diffraction (LEED) [10], scanning tunneling microscopy (STM) [11, 12, 13, 7], surface x-ray diffraction (SXRD) [14] and ion scattering [15]. There have also been a large number of first principles theoretical studies $[16,17,18,19,20]$. Early contributions included a periodic Hartree Fock study [16] within a linear combination of atomic orbitals (LCAO) formalism and a density functional theory study (DFT) which used a full potential linear augmented plane wave (FP-LAPW) method [17]. In each case the model systems studied were rather small and the surfaces only partially relaxed. More recently plane-wave $(\mathrm{PW})$ pseudopotential calculations have been used based on both the local density (LDA) and generalised gradient (GGA) approximations to DFT $[18,19,20]$. These calculations included extensive relaxations of the surface structure and were based on larger structural models. Despite these extensive efforts the agreement between computed and measured structures 
is semi-quantitative at best. The extensive experience of calculations on bulk oxides which has been built up in recent years leads one to expect that DFT and HF calculations will reproduce experimental bond lengths to somewhat better than $0.1 \AA$. At the (110) surface the inward relaxation of the bridging oxygen ion determined by SXRD is $-0.27 \AA$ while most calculations find a relaxation of less than $-0.1 \AA$. The current article is concerned with a detailed examination of this discrepancy.

Transition metal oxides represent a significant challenge to first principles calculations. The localised nature of the oxygen $2 p$ and in particular the titanium $3 d$ states makes the PW pseudopotential method particularly demanding. The expansion of localised orbitals in plane waves requires large kinetic energy cut-offs to converge the total energy. In addition the separation of the $s p$ and $d$ electron eigenvalues in the periodic system is dependent on the choice of the atomic reference state from which the pseudopotential is constructed. Great care must be taken to understand the effect of these approximations on computed material properties. Recently, Hamann [21] has discussed in detail calculations for bulk $\mathrm{TiO}_{2}$ and concluded that computed geometries and energies varied significantly with the choice of the local component in the pseudopotential.

With these difficulties in mind we have chosen to use two complementary all electron techniques to study the $\mathrm{TiO}_{2}$ (110) surface. Firstly the FP-LAPW method [22] employs a basis set consisting of plane waves which, (inside atom-centered, non-overlapping spheres) are matched continuously in value and slope to an expansion in terms of spherical harmonics (here up to $l_{\max }^{\mathrm{wf}}=10$ ) and numerical solutions of the radial Schrödingerequation. This basis set has maximum flexibility and ensures the high accuracy of the calculations. Secondly, the LCAO method which employs a basis set of atom centred Gaussian functions for which an hierachy of basis sets approaching complete convergence has recently been developed and tested for $\mathrm{TiO}_{2}$ surfaces [23, 24]. By confining all numerical approximations we provide definitive DFT-GGA results for this surface. Using the energy surface obtained we are able to examine the comparison of theory and experiment and thus resolve this long standing problem. 
The next section contains details of the computational methods used, the results are then presented and discussed and our conclusions are summarised in the final section.

\section{Methodology}

In this section we give details of the structural model used to describe the (110) surface and of the FP-LAPW and LCAO methods used to perform our calculations.

\section{$2.1 \quad$ Structural Model}

The (110) surface is modelled as a slab periodic in [001] and [110] directions but finite in the [110] direction (as shown in figure 1). An essential requirement for quantitative studies is that computed properties are fully converged with respect to the thickness of the slab. A systematic series of tests in which the structures of slabs of varying thickness were fully relaxed revealed that for a slab containing 21 atomic layers (ie: $7 \mathrm{O}-\mathrm{Ti}_{2} \mathrm{O}_{2}$-O layers) the surface energy was converged to better than $0.1 \mathrm{Jm}^{-2}\left(6 \mathrm{eV}^{-2}\right)$ and geometric displacements to better than $0.02 \AA[23,24]$.

\subsection{FP-LAPW}

In the FP-LAPW calculations a supercell periodic in 3 dimensions was used in which the slab geometry described above was repeated in the [110] direction with slabs separated by a large vacuum region of $9 \AA$ to ensure that there were no significant interactions between the slabs. The size of this structural model is significantly larger than that used in previous studies of surface structures within the FP-LAPW method. These calculations have been made feasible by recent developments and improvements of the method [25].

In order to minimise the number of k-points required to converge the surface energy care was taken to ensure a systematic cancellation of errors between the calculations on the slab and bulk crystal. 
This is achieved by describing the bulk crystal with a unit cell corresponding as closely as possible to that used to describe the surface and using identical computational parameters in both sets of calculations. A bulk unit cell with six times the volume of the primitive cell was used. With this arrangement we found that a uniform k-point mesh with three points in the irreducible part of the Brillouin zone was adequate. A kinetic-energy cutoff for the plane-wave basis of $E_{\max }^{\mathrm{wf}}=22$ Ry was used. This is a rather high value for such huge systems. However, because of the large surface relaxations we had to use rather small muffin-tin spheres $\left(R_{\mathrm{Ti}}^{\mathrm{MT}}=0.90 \AA R_{\mathrm{O}}^{\mathrm{MT}}=0.80 \AA\right.$ ,) and therefore a large value for $E_{\max }^{\mathrm{wf}}$ was mandatory to ensure good numerical accuracy. The electron density and potential are expanded in lattice harmonics up to $l_{\max }^{\mathrm{pot}}=6$ inside the spheres, and the wave functions are expanded in angular momenta up to $l_{\max }^{\mathrm{wf}}=10$. The electron density and potential in the interstitial region are expanded in plane waves up to $144 \mathrm{Ry}$. The core states are treated fully relativistically. The Ti $3 s, 3 p$ and $\mathrm{O} 2 s$, which are represented by local orbitals, as well as the valence states ( $\mathrm{Ti} 3 d, 4 s, \mathrm{O} 2 p)$ are treated scalar-relativistically.

The relaxations of all atoms in the slab were considered, and the surface structure was determined by relaxing the entire system to equilibrium. All the atoms were relaxed according to the force directions and total energy minimization until all atom forces for a geometry fall below a certain limit. The process of the structure optimization has been described in reference [26].

\section{$2.3 \quad$ LCAO}

The LCAO calculations were performed with the CRYSTAL program [27]. In contrast to the FP-LAPW calculations the slab geometry was modelled as periodic in two dimensions and finite in the third removing the need to define a vacuum gap.

The main approximation in the LCAO formalism is the choice of the local basis set used to expand the Bloch orbitals of the crystal. The basis set is made up of atom centred Gaussian functions with $s, p$ or $d$ symmetry. A systematic hierachy of basis sets was developed in a recent study of the $\mathrm{TiO}_{2}$ (100) surface [23]. In this study it was shown that sets employing two basis functions to 
describe the valence electrons (so called, double valence - DV) can predict surface ionic relaxations to an accuracy of $0.02 \AA$ compared to the basis set limit. Tests for the (110) surface confirm these conclusions and so in the current study a DV basis set has been used the details of which are given elsewhere $[23,24]$.

The total energy of the bulk crystal and surface were explicitly converged with respect to k-point sampling. A Pack-Monkhorst mesh [27, 28] of order 4 which yields $10 \mathrm{k}$-points in the irreducible Brillouin zone of a (110) slab and 36 in that of the bulk crystal were used. This procedure of converging the bulk and slab energies explicitly with respect to $\mathbf{k}$-space sampling removes the reliance on a systematic cancellation of errors when computing surface properties.

CRYSTAL computes matrix elements of the Coulomb, exchange and correlation matrix elements by direct summation over the infinite periodic lattice. Very efficient computational schemes for truncating the lattice summations have been developed [29]. The accuracy of the summation is based on overlap criteria for the atomic orbitals. Details of the control of these criteria have been described elsewhere $[30,31,32,23]$. In the current study the criteria were chosen to achieve an accuracy in the relative energies of the surface and bulk structures of order $1 \mathrm{meV}$ per cell [33].

The surface relaxations were performed using an adapted conjugate gradient minimisation algorithm [34] to a tolerance of $0.01 \AA$ in atomic positions and $10^{-5} \mathrm{eV}$ in the total energy.

\subsection{The Exchange-Correlation Functional}

The main results of this article have been computed using the GGA functionals recently introduced by Perdew, Burke and Ernzerhof (PBE) [35]. In addition a number of alternative treatments of the electron exchange and correlation interactions have been used in order to establish the sensitivity of key results. Within the LCAO formalism Hartree-Fock (ie: non-local exchange with no treatment of correlation) and LDA calculations were also performed [36, 37, 24]. Within the FP-LAPW formalism the GGA functional proposed by Perdew and Wang (PWGGA) [38] was also used. 
We find that the structural properties of the bulk crystal and the surface are very insensitive to the choice of functional. The PBE and PWGGA approaches agree to well within the numerical tolerances and differences between HF, LDA and GGA are confined to less than $0.02 \AA$ in any surface or bulk displacement [24]. It is likely that differences between previous calculations which have been assigned to differing treatments of exchange and correlation are in fact due to incomplete convergence of the calculations $[16,17,18,19,20]$.

\section{Results and Discussion}

The atomistic structure of the $\mathrm{TiO}_{2}(110)$ surface is depicted in figure 1 in which labels are assigned to the atoms in the surface region. The relaxations of the top few layers computed here and in a number of recent studies are compared to those deduced from surface x-ray diffraction experiments in table 1.

At first sight the most notable feature of this data is that the agreement between theory and experiment is poor. This is particularly true for the position of the bridging oxygen ion $\left(\mathrm{O}_{(3)}\right)$ for which the computed relaxation is never more that $-0.16 \AA$ while the experiment finds $-0.27 \AA$ . On closer examination it is the discepancy between the various theoretical approaches which gives most cause for concern. This is especially true for the current study in which, as stated above, great care has been taken to control the effects of all numerical tolerances on two different all-electron approaches. Nevertheless the relaxation of the bridging oxygen ion is computed to be -0.02 or $-0.16 \AA$ and the relaxation of the six fold coordinated Ti-ion directly "beneath" it $\left(\operatorname{Ti}_{(1)}\right)$ to be 0.23 or $0.08 \AA$ in the LCAO and FP-LAPW methods respectively. These variations are significantly larger than the numerical errors that one would expect. It is however evident that the Ti-O separation in the [110] direction is more consistent (this is reported in the final row of table 1). In the current study this is $1.04 \AA$ and $1.03 \AA$ in the FP-LAPW and LCAO calculations respectively while $0.89 \pm 0.13 \AA$ is deduced from the SXRD experiment and ion scattering measurements [15]. 
This observation leads one to the hypothesis that the energy surface with respect to vertical displacement of the bridging oxygen is rather flat and that the displacement involves a cooperative motion of the surface ions which, at the least, involves the six fold coordinated surface Ti-ion $\left(\mathrm{Ti}_{(1)}\right)$

In order to explore this possibility a number of relaxations have been performed in which the position of the bridging oxygen ion has been constrained but the surrounding surface ions fully relaxed. For reasons of efficiency these calculations were performed on a relatively small system containing 9 atomic layers. The minimum energy structure of this system is is somewhat displaced from that of larger systems but it displays all of the features necessary to examine the qualitative structure of the energy surface. The resultant energy surfaces computed within both the FP-LAPW and LCAO formalisms are displayed in figure 2. The LCAO calculations on this smaller system were performed with a very large basis set (the TVAEd basis set reported in reference [23, 24]). In order to give some feeling for the energy scale a line representing a typical room temperature thermal energy per degree of freedom $\left(k_{B} T=0.025 \mathrm{eV}\right)$ has been drawn on the figure. The energy surface is sufficiently flat for thermal vibrations to leave the minimum undetermined to about $0.15 \AA$. From this it is clear that the discrepancy between different theoretical approaches is due to the difficulty in finding an absolute energy minimum in this very flat energy surface.

We may associate the flat energy surface with a highly anisotropic and anharmonic surface vibrational mode. The nature of the mode is easily seen from an animation of the atomic positions. The displacements explored at thermal energies approximately corresponding to room temperature are displayed in figure 3. During this vibration the $\mathrm{O}_{(3)}-\mathrm{Ti}_{(1)}$ separation along [110] remains very close to $1.03 \AA$ and the separation of $\mathrm{Ti}_{(1)}$ and $\mathrm{O}_{(6)}$ is also nearly constant. The displacements of $\mathrm{Ti}_{(2)}$, $\mathrm{O}_{(4)}$ and $\mathrm{O}_{(5)}$ are very small. Thus, to the first approximation, we may understand the vibration as a "rigid unit mode" of the square planar $\mathrm{TiO}_{4}$ unit containing $\mathrm{Ti}_{(1)}$ and its four nearest neighbours - $\mathrm{O}_{(3)}, \mathrm{O}_{(6)}$ and their periodic images (this is depicted in the lower left panel of figure 3).

An immediate consequence of rigid unit mode is that the structure of the $\mathrm{TiO}_{2}(110)$ surface 
apparent in the experimental probes applied at finite temperature does not correspond to the minimum energy configuration computed within a total energy calculation. In order to make such a comparison a more complete treatment of the effect of surface vibrational modes on our interpretation of experimental data must be explored. In the case of SXRD and LEED experiments this necessitates the modelling of an anharmonic thermal vibration which is also highly anisotropic. The current practice is to fit the diffraction rods within an harmonic and often isotropic DebyeWaller model which is inadequate for the current case. A quantitative interpretation of STM images will require a treatment of the tip-surface interaction as this is likely to result in significant distortions of the surface structure.

In addition the free energy associated with soft surface modes cannot be neglected. This has been demonstrated in recent first principles free energy calculations on the $\mathrm{Ag}(111)$ surface. In this system the minimum energy structure corresponds to a contraction of the outer layer spacing of $-1.0 \%$ while the free energy minimum at $1150 \mathrm{~K}$ yields an expansion of $6.3 \%$ - a shift in the interlayer spacing of $0.16 \AA$ [39]. We expect a significantly larger effect at the $\mathrm{TiO}_{2}(110)$ surface due to the presence of a soft, anharmonic, surface vibrational mode.

\section{Conclusion}

There is poor quantitative agreement between the structures predicted from the minimum energy configuration of first principles calculations and those deduced from x-ray diffraction experiments for the $\mathrm{TiO}_{2}$ (110) surface. We find that a very soft and anharmonic surface rigid-unit vibrational mode involves displacements of the surface ions of approximately $0.15 \AA$ for thermal vibrations corresponding to room temperature. In order to perform an accurate comparison between theory and experiment for this and perhaps other surfaces it will be necessary to take account of such anisotropic vibrations in models used to interpret experimental data. In addition the contribution of the vibrational entropy to the surface free energy is likely to be significant and must be taken 
into account when computing surfaces energies and structures. 


\section{References}

[1] V.E. Henrich and A.F. Cox. The Surface Science of Metal Oxides. Cambridge University Press, Cambridge, 1993.

[2] A. Fujishima and K. Honda. Nature, 238:37, 1972.

[3] New Scientist. 8 August:8, 1998.

[4] New Scientist. 13 September:15, 1997.

[5] New Scientist. 10 October:11, 1998.

[6] I.M. Butterfield, P.A. Christensen, A. Hamnett, K.E. Shaw, G.M. Walker, S.A. Walker, C.R. Howarth. J. App. Electrochem., 27:385, 1997.

[7] R.A. Bennett, P. Stone, N.J. Price and M. Bowker. Phys. Rev. Lett., 82:3831, 1999.

[8] J. Muscat, N.M. Harrison and G. Thornton. Phys. Rev. B, 59:15457, 1999.

[9] A.T. Paxton and L. Thien-Nga. Phys. Rev. B, 57:1579, 1998.

[10] V.E. Henrich and R.L. Kurtz. Phys. Rev. B, 23:6280, 1981.

[11] H. Onishi and Y. Iwasawa. Surf. Sci., 313:L783, 1994.

[12] P.W. Murray, N.G. Condon and G. Thornton. Phys. Rev. B, 51:10989, 1995.

[13] U. Diebold and J.F. Anderson and K.O. Ng and D. Vanderbilt. Phys. Rev. Lett., 77:1322, 1996.

[14] G. Charlton, P.B. Howes, C.L. Nicklin, P. Steadman, J.S.G. Taylor, C.A. Muryn, S.P. Harte, J. Mercer, R. McGrath, D. Norman, T.S. Turner and G. Thornton. Phys. Rev. Lett., 78:495, 1997.

[15] B. Hird, R.A. Armstrong. Surf. Sci. Lett., 385:L1023-L1028, 1997. 
[16] P. Reinhardt and B.A. Hess. Phys. Rev. B, 50:12015, 1994.

[17] D. Vogtenhuber, R. Podloucky, A. Neckel, S.G. Steinemann and A.J. Freeman. Phys. Rev. B, 49:2099, 1994.

[18] M. Ramamoorthy, D. Vanderbilt and R.D. King-Smith. Phys. Rev. B, 49:16721, 1994.

[19] P.J.D. Lindan, N.M. Harrison, M.J. Gillan and J.A. White. Phys. Rev. B, 55:15919, 1997.

[20] S.P. Bates, G. Kresse, M.J. Gillan. Surf. Sci., 385:386-394, 1997.

[21] D.R. Hamann. Phys. Rev. B, 56:14979, 1997.

[22] P. Blaha, K. Schwarz, P. Sorantin, and S.B. Trickey. Comp. Phys. Commun., 59:399, 1990.

[23] J. Muscat, N.M. Harrison and G. Thornton. Phys. Rev. B, 59:2320, 1999.

[24] J. Muscat. The Phase Stability, Surface Structure and Defect Chemistry of Titanium Dioxide from First Principles. PhD thesis, Department of Chemistry, University of Manchester, 1999.

[25] M. Petersen, F. Wagner, L. Hufnagel, M. Scheffler, P. Blaha and K. Schwarz. Comp. Phys. Commun. in press., 1999.

[26] B. Kohler, S. Wilke, M. Scheffler, R. Kouba, and C. Ambrosch-Draxl. Comput. Phys. Commun., 94:31, 1996.

[27] R. Dovesi, V.R. Saunders, C. Roetti, M. Causà, N.M. Harrison, R. Orlando and E. Aprà. CRYSTAL95 User's Manual. University of Turin, Turin, 1996.

[28] J.D. Pack and H.J. Monkhorst. Phys. Rev. B, 16:1748, 1977.

[29] C. Pisani, R. Dovesi and C. Roetti. Hartree-Fock Ab Initio Treatment of Crystaline Systems, volume 48. Springer-Verlag, Berlin, 1988.

[30] R. Orlando, R. Dovesi, C. Roetti and V.R. Saunders. J. Phys. Cond. Matt., 2:7769, 1990. 
[31] R. Dovesi, M. Causa, R. Orlando, C. Roetti and V.R. Saunders. J. Chem. Phys., 92:7402, 1990.

[32] C. Pisani (Ed.). Quantum Mechanical Ab-initio Calculation of the Properties of Crystaline Materials, volume 67. Springer-Verlag, Berlin, 1996.

[33] Explicitly the ITOL parameters were set to $10^{-6}, 10^{-6}, 10^{-6}, 10^{-6}$ and $10^{-12}$.

[34] C. Zhu, R.H. Byrd, P. LU, J. Nocedal. L-BFGS-B - Fortran Subroutines for Large Scale Bound Constrained Optimisation. Dept of Elec. Eng. and Comp. Sci, Northwestern University, Illinois, 1994.

[35] J.P. Perdew and K. Burke and M. Ernzerhof. ACS Symposium series, 629:453, 1996.

[36] P.A.M. Dirac. Proc. Cam. Phil. Soc., 26:376, 1930.

[37] J.P. Perdew and A. Zunger. Phys. Rev. B, 23:5048, 1981.

[38] J. P. Perdew, J. A. Chevary, S. H. Vosko, K. A. Jackson, M. R. Pederson, D. J. Singh, and C. Fiolhais. Phys. Rev. B, 46:6671, 1992.

[39] J. Xie, S. Gironcoli, S. Baroni and M. Scheffler. Phys. Rev. B, 59:970, 1999. 


\begin{tabular}{|c|c|c|c|c|c|c|c|c|c|c|c|c|}
\hline \multirow{3}{*}{$\begin{array}{l}\text { Label } \\
\operatorname{Ti}_{(1)}\end{array}$} & \multicolumn{2}{|c|}{ FP-LAPW-PBE } & \multicolumn{2}{|c|}{ LCAO-PBE } & \multicolumn{2}{|c|}{ PW-LDA [18] } & \multicolumn{2}{|c|}{ PW-GGA [20] } & \multicolumn{4}{|c|}{ SXRD [14] } \\
\hline & {$[110]$} & {$[110]$} & {$[110]$} & [110] & {$[110]$} & {$[110]$} & {$[110]$} & [110] & & 10] & & 10] \\
\hline & 0.08 & 0.00 & 0.23 & 0.00 & 0.13 & - & 0.23 & 0.00 & 0.12 & \pm 0.05 & 0.00 & - \\
\hline $\operatorname{Ti}_{(2)}$ & -0.23 & 0.00 & -0.17 & 0.00 & -0.17 & - & -0.11 & 0.00 & -0.16 & \pm 0.05 & 0.00 & - \\
\hline $\mathrm{O}_{(3)}$ & -0.16 & 0.00 & -0.02 & 0.00 & -0.06 & - & -0.02 & 0.00 & -0.27 & \pm 0.08 & 0.00 & - \\
\hline $\mathrm{O}_{(4)}$ & 0.09 & -0.06 & 0.13 & -0.05 & 0.13 & -0.04 & 0.18 & -0.05 & 0.05 & \pm 0.05 & -0.16 & \pm 0.08 \\
\hline $\mathrm{O}_{(5)}$ & 0.09 & 0.06 & 0.13 & 0.05 & 0.13 & 0.04 & 0.18 & 0.05 & 0.05 & \pm 0.05 & 0.16 & \pm 0.08 \\
\hline $\mathrm{O}_{(6)}$ & -0.09 & 0.00 & 0.02 & 0.00 & -0.07 & - & 0.03 & - & 0.05 & \pm 0.08 & 0.00 & - \\
\hline $\mathrm{Ti}_{(7)}$ & 0.07 & 0.00 & 0.14 & 0.00 & 0.06 & - & 0.12 & - & 0.07 & \pm 0.04 & 0.00 & - \\
\hline $\operatorname{Ti}_{(8)}$ & -0.13 & 0.00 & -0.10 & 0.00 & -0.08 & - & -0.06 & - & -0.09 & \pm 0.04 & 0.00 & - \\
\hline $\mathrm{O}_{(9)}$ & -0.05 & 0.00 & 0.00 & 0.00 & 0.02 & - & 0.03 & - & 0.00 & \pm 0.08 & 0.00 & - \\
\hline $\mathrm{O}_{(10)}$ & -0.04 & 0.03 & 0.03 & 0.03 & -0.03 & 0.05 & 0.00 & 0.02 & 0.02 & \pm 0.06 & 0.07 & \pm 0.06 \\
\hline $\mathrm{O}_{(11)}$ & -0.04 & -0.03 & 0.03 & -0.03 & -0.03 & -0.05 & 0.00 & -0.02 & 0.02 & \pm 0.06 & -0.07 & \pm 0.06 \\
\hline $\mathrm{O}_{(12)}$ & -0.04 & 0.00 & -0.01 & 0.00 & -0.01 & - & 0.03 & - & -0.09 & \pm 0.08 & 0.00 & - \\
\hline $\operatorname{Ti}_{(13)}$ & 0.02 & 0.00 & 0.05 & 0.00 & - & - & - & - & - & - & - & - \\
\hline $\mathrm{Ti}_{(14)}$ & -0.08 & 0.00 & -0.06 & 0.00 & - & - & - & - & - & - & - & - \\
\hline $\mathrm{O}_{(15)}$ & -0.07 & 0.00 & 0.01 & 0.00 & - & - & - & - & -0.12 & \pm 0.07 & 0.00 & - \\
\hline $\mathrm{O}_{(16)}$ & -0.03 & 0.02 & 0.01 & -0.02 & - & - & - & - & - & - & - & - \\
\hline $\mathrm{O}_{(17)}$ & -0.03 & -0.02 & 0.01 & 0.02 & - & - & - & - & - & - & - & - \\
\hline $\mathrm{O}_{(18)}$ & -0.02 & -0.02 & 0.01 & 0.00 & - & - & - & - & - & - & - & - \\
\hline $\mathrm{O}_{(19)}$ & 0.02 & -0.02 & -0.01 & 0.00 & - & - & - & - & - & - & - & - \\
\hline $\mathrm{O}-\mathrm{Ti}$ & & & & & & & & & & 0.89 & $=0.13$ & \\
\hline
\end{tabular}

Table 1: The displacements (in $\AA$, from bulk terminated positions) of the ions at the (110) $(1 \times 1)$ surface computed within the FP-LAPW and LCAO formalisms compared to previous plane wave calculations and those deduced from surface x-ray diffraction data. The last row gives the $\mathrm{O}_{(3)}-\mathrm{Ti}_{(1)}$ separation in the [110] direction. The labels and directions refer to figure 1. 


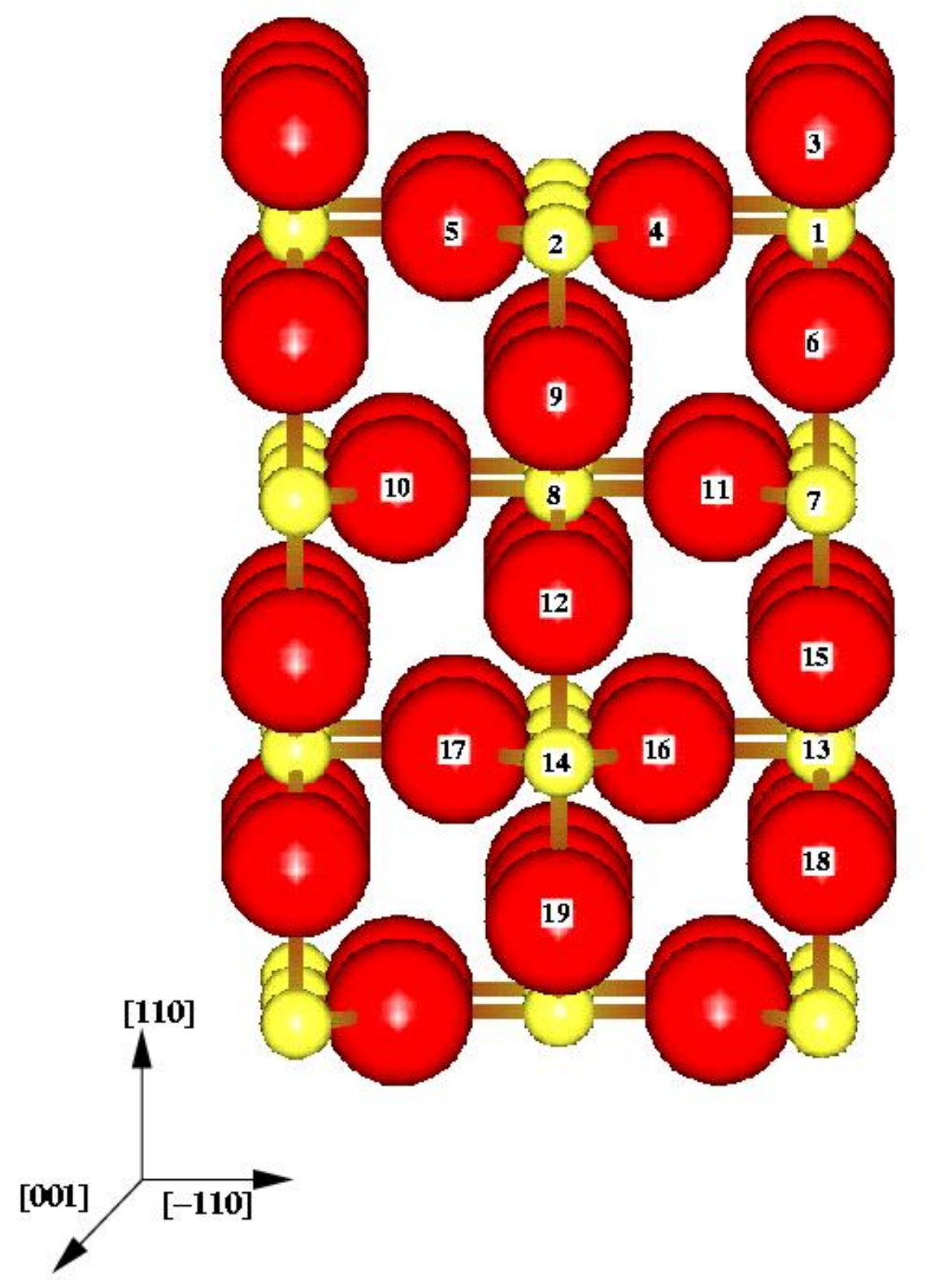

Figure 1: A section through a (110) surface viewed in the [001] direction. The surface is based on a 21 layer slab with a mirror plane through the centre of the slab. All symmetry inequivalent ions in the top half of the slab are labelled. 


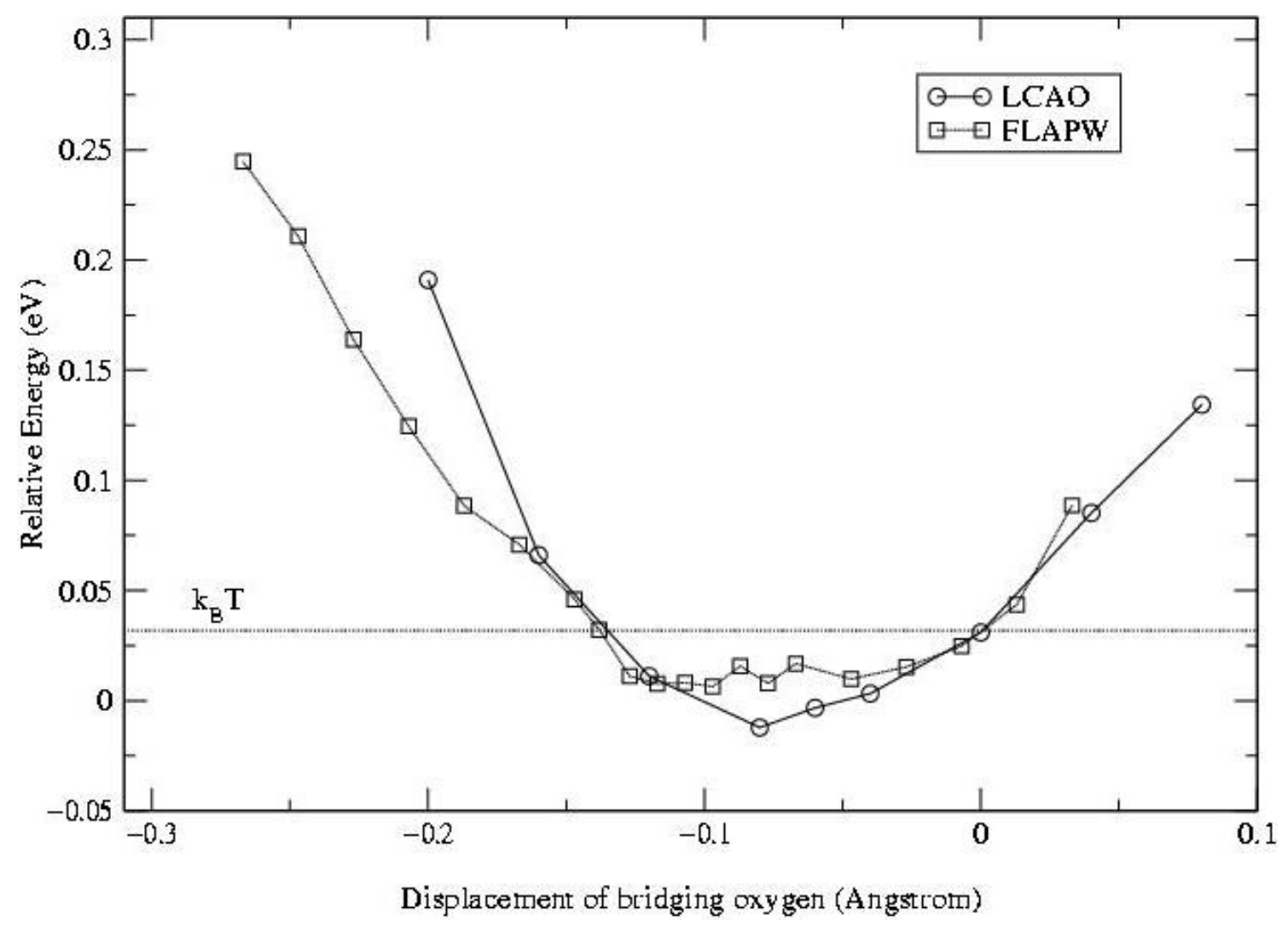

Figure 2: The relative energy of the (110) surface computed within the LCAO and FP-LAPW formalisms for various fixed positions of the bridging oxygen ion relative to the unrelaxed bulk terminated position.

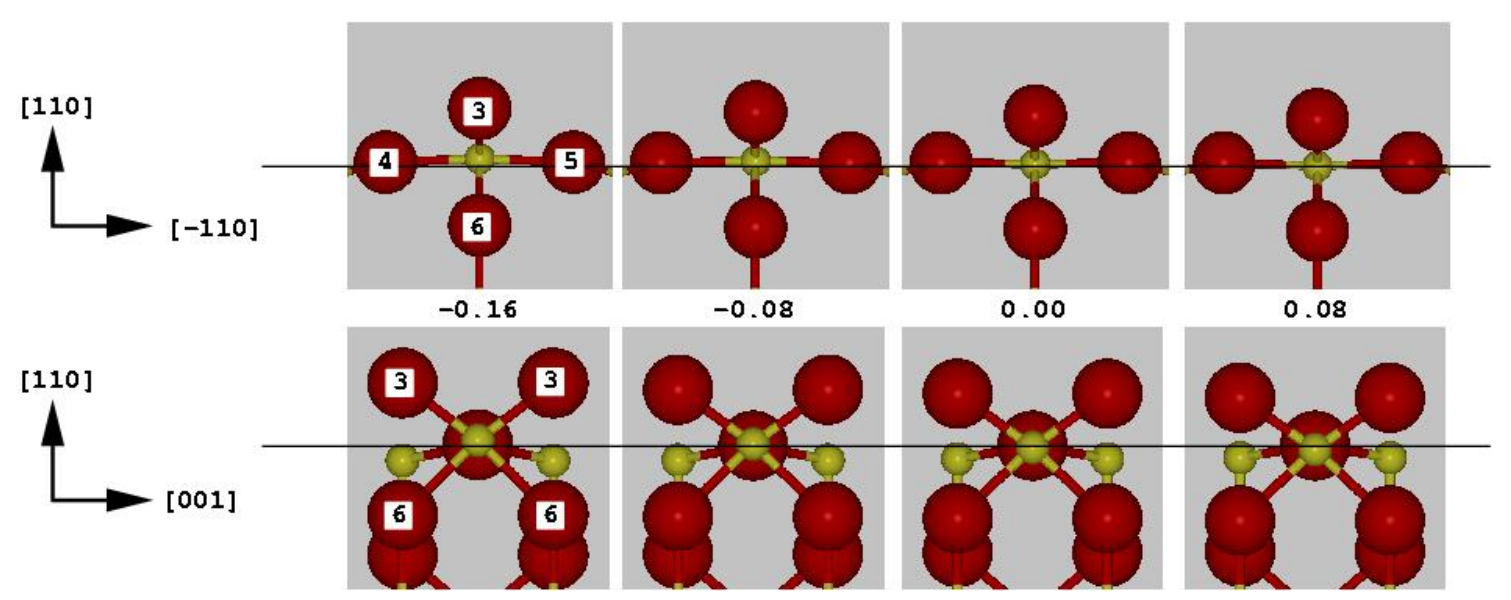

Figure 3: The approximate room temperature thermal motions of the atoms within the soft rigid unit mode of the (110) surface. The atom labels correspond to those in figure 1.

The oxygen atoms comprising the rigid unit are labelled in the lower left panel. 